\title{
Jahres-Inhalt, Vol. 6, No. 6, 1983
}

Bernsmeier, R.; Holzgrebe, E.; Luck, P.; Bruhn, H. D. und Zurborn, K. H. , Kiel

Thrombin, Faktor XIII und Fibronectin als Regulatoren der Proliferation von Tumorzellen und Gefäßwandzellen 4

Dimitriadis, K.; Boutís, L.; Tavantzis, J.;

Mouratidou, D.; Bouchoris, N.; Koukourikos, S.;

Hatzigogos, K. und Safarini, S.,

ThessalonikilGriechenland

Chemotherapie des nicht-kleinzeiligen Bronchialkarzi-

noms 8

Blum, U.; Ungeheuer, E. und Wacha, H., Frankfurt/M.

Bedeutung der klinischen Symptomatik für die Früh-

erkennung des Bronchialkarzinoms 12

Schütte, J.; Bremer, K.; Niederle, N.; Schoetensack, B.; Schmidt, C. G. und Seeber, S., Essen

Sequentiell-alternierende Chemotherapie nicht-semino-matöser Hodentumoren mit

Adriamycin/Cisplatin und Bleomycin/Vinblastin - Therapieansprechen und -versa-gen in

Abhängigkeit von Histologie und Tumorstadium -. 16

Steinke, B.; Voss, A. C; Ostendorf, P. und

Waller, H. D., Tubingen

Kombinierte Chemo- und Radiotherapie des kleinzelli-

gen Bronchialkarzinoms mit Anwendung hoher Strahlen-

dosen 22

Winkler, K.; Beron, G.; Phone, L; Müller-Wickup, J. und Landbeck, G., Hamburg, Jürgens, H. und Goebel, U., Düsseldorf, Gutjahr, P., Mainz, Spaar, H.-J., Bremen, Drescher, J., Oldenburg und Thomas, P., Krefeld

Therapie der akuten Lymphoblastenleukämie im Kindes-

alter - Multizentrische prospektive Therapiestudie

COALL-80 26

Neuigkeiten für die klinische Praxis 34

Gericke, D., Frankfurt, Hofstetter, A.; Keiditsch, E. und Lersch, Chr., München

Möglichkeiten immunologischer Beeinflussung des

Brown-Pearce-Karzinoms am Kaninchen 36

Buchbesprechungen 44

Onkologie-Mitteilungen

König, H. J.; Krähling, K. H. und Brandt, M., Münster

Stellenwert der Hypophysektomie beim metastasieren-

den Mammakarzinom 56

Mende, S.; Bleichner, F.; Stoeter, P. und Meuret, G.,

Ravensburg

Erfolgreiche Behandlung von Hirnmetastasen bei 
Mamma-Karzinom mit nicht liquorgängigen Zytostatika

und Hormonen

58

Weber, W.; Obrecht, J. P. und Germano, G., Basel, Arnold, H., Freiburgi.Br., Drings, P.,

Heidelberg, Mutter, K. M., Münster, Queisser, W., Mannheim und Westerhausen, M., Duisburg

Etretinat (Ro 10-9359, Tigason) + CCNU (1-(2-chloro-

athyl)-3-cyclohexyl-1-nitrosourea) + Bleomycin versus

$\mathrm{CCNU}+$ Bleomycin in der Behandlung des fortgeschrit-

tenen Plattenepithel-Karzinoms des Bronchus (AlO-Stu-

die BS 1/78) 62

Boll, I., Berlin

Unterschiedliche Wirkungen von Zytostatika auf die

Zellkinetik des normalen und leukämischen mensch-

lichen Knochenmarks in vitro 66

Aigner, K.; Tonn, J. C. und Hechtel, R., Gießen und Seuffer, R., Reutlingen

Die intraarterielle Zytostatikatherapie mit venöser Filtra

tion im halboffenen System 74

Neuigkeiten für die klinische Praxis 78

Aulbert, E. und Schmidt, C. G., Essen

Anaphylaktische Reaktion unter Cyclophosphamid-Infu-

sion 82

Kehl, A.; Bergholz, M.; von Hey den, H. W. und

Nagel, G. A., Göttingen

Toxisch-allergisches Lungenödem nach Cyclophospha-

mid-und Ifosfamid-Therapie. - Ein Fallbericht 84

von Hey den, H. W.; Scherff A.; Nagel, G. A.; Gahr, M. und Schröter, W., Göttingen

Erfahrungen mit der Sedimentationsleukapharese nach

Djerassi 88

Onkologie-Mitteilungen 95

H. G. Mertens

Editorial 106

V. Schöck, Bielefeld-Bethel

Hochdosierte intralumbale Methotrexatgaben

Schroder, M.; von Hey den, H. W.; Scherpe, A.;

Borghardt, J.; Beyer, J.-H. und Nagel, G. A., Göttingen,

Gerhartz, H. und Kastenbauer, E., Berlin,

Westerhausen, M. und Schroeder, M. , Duisburg,

Caliebe, W.; Rudert, H. und Lissers, R., Kiel,

Hofmann, J. und Schneider, B., Hannover

Zweite Zwischenauswertung einer Chemotherapiestudie

an Patienten mit Plattenepithelkarzinomen des Kopf-

Hals-Bereiches. - Vergleich zweier Therapieregime: Cis-

Diaminodichlorplatinum-(П) und Bleomycin gegen

Methotrexat und Vindesin 114

Krebs, A. und Franke, H., Würzburg

Die Steigerung der Serumkonzentration von a- und B-

Globulinen und speziell des karzinoembryonalen Anti 
gens durch Ultraschallbestrahlung von Lebergeweben

bzw. von CEA-aktiven Karzinomen 121

Klippel, K. F. und Alves de Oliveira, C. R., Celle,

Axt, D.; Schärfe, T. und Preiss, J., Mainz

Erhöhte CEA-Werte nach Zystektomie wegen Harnbla-

senkarzinom und intestinaler Harnableitung nicht bewei-

send für Tumorrezidiv126

Buchbesprechungen 132

Pfeilsticker, U.; Heidemann, E.; Wilms, K.; Voss, A. C. und Waller, H. D., Tubingen

Vergleich der Therapieergebnisse bei der Behandlung der chronischen Myelose mit

Milzbestrahlung oder Busulfan . 136

Neuigkeiten für die klinische Praxis 140

Steinke, B.; Heidemann, E.; Ostendorf, P.; Wilms, K. und Waller, H. D., Tubingen

Chemotherapie nicht-seminomatöser Hodentumoren. -

Ergebnisse einer Pilot-Studie mit reduzierter Cis-Platin-

Dosis 144

Polterauer, P.; Meisl, F.; Funovics, J. und Holzner, J. H.,

WienlÖsterreich

Frühkarzinom des Magens. - Verlaufsbeobachtungen

über23Monate.EinFallbericht 148

Onkologie-Mitteilungen $\quad 154$

Hellriegel, K. P. , Berlin

Editorial 169

Hoelzer, D., Ulm, etal.

Patientenrekrutierung und Ergebnisse einer Vorphase-

studie zur Therapie der akuten lymphatischen Leukämie

und der akuten undifferenzierten Leukämie des Erwach-

senen 170

Büchner, Th.; Hiddemann, W.; Urbanitz, D.;

Kamanabroo, D.; Schulte, H.

und van de Loo, J. , Münster

Die Studie Münster über intensivierte Induktions- und

Konsolidierungstherapie ohne Erhaltungstherapie der

AML. - Resultate bei 93 Patienten - zelluläre Determi-

nanten für Response und Remissionsdauer 176

Hiddemann, W.; Büchner, Th.

undWörmann, B., Münster; Andreeff, M.;Arlin, Z.

und Clarkson, B. D., New York

Vergleich der Therapieeffektivität von zwei Induktions-

protokollen bei akuter myeloischer Leukämie (AML)

mittels exakter Quantifizierung der Knochenmarkzellula-

rität 179

Nowrousian, M.R.; Kubaschinski, G.; Hossfeld, D.K.;

Schaefer, U. W. und Schmidt, C. G., Essen

Ergebnisse der Behandlung der akuten myeloischen

Leukämie beim Erwachsenen 184 
Fiebig, H.H.; Henß, H. und Engelhardt, R., Freiburg;

Hoelzer, D. , Ulm; Pralle, H. , Gießen;

Link, H., Tubingen und Schmitz, R., Kiel

Phase-II-Studie von AMSA bei Erwachsenen mit therapierefraktären akuten Leukämien 188

Mitrou, P. S., Frankfurt

Therapieergebnisse mit Aclacinomycin A bei rezidivierten akuten Leukämien 192

Buchbesprechungen 195

Neuigkeiten für die klinische Praxis 198

Sonderbände 199

Willemze, R.; Fibbe, W.E.

und Zwaan, F. E. , Leiden/Niederlande

Erfahrungen mit mittel- und hochdosiertem Cytosinara-

binosid bei refraktärer Leukämie 200

Kleinmanns, G. und Westerhausen, M., Duisburg;

Dauer, W. und Knief, J.J., Wesel;

Therapie-Ergebnisse beim fortgeschrittenen Ovarialkar-

zinom 206

Vorwort 215

Referate-Themen 216

Abstracts zum Hämatologen-Kongreß Münster,

Oktober $1983 \ldots 217$

Neuigkeiten für die klinische Praxis

254

Onkologie-Mitteilungen

Buchbesprechungen 278

Impressum 279

Aul, C.; Fischer, J. Th. und Schneider, W., Düsseldorf

Diagnostik der akuten myeloischen Leukämien $\quad 280$

Illiger, H. J.; Peiss, J.; Vaupel, H. A. und Hartlapp, J. H., Bonn

Vinblastin, 5-Fluorouracil und Prednison (VFP) als

»second-line «-Chemotherapie. - Ein Beitrag zum Pro

blem der optimalen Therapiesequenz beim metastasier-

ten Mammakarzinom 288

Knispel, J.; Thiel, R. und Wallis, H., Hamburg

Therapieabbruch bei 3 Osteosarkom-Patienten. - Eine

Kasuistik 292

Schulz, A.; Fischer, H.-P.; Breithaupt, H. und

Pralle, H., Gießen

Therapie-Response verschiedener histologischer Sub-

typen des Osteosarkoms unter hochdosierter Metho-

trexat-Behandlung 296

Klippstein, Th. und Mitrou, P. S., Frankfurt;

Graubner, M., Gießen; Berdel, W. E., München;

Mende, S., Ravensburg; Gropp, C, Marburg, und

Diehl, V., Hannover 
Ergebnisse der Chemotherapie inoperabler nicht-kleinzelliger Bronchialkarzinome mit VP 16-213 (Etoposid) und Cis-Platin.- Eine Phase-II-Studie 306

Neuigkeiten für die klinische Praxis 312

Bichler, E. und Daxecker, F., Innsbruck

Zur Dunkeladaptation bei Patienten mit Tumoren im Kopf-Hals-Bereich 314

Platz, H. und Fries, R., Linz, Hudec, M., Wien

Retrospektive DÖSAK-Studie über Karzinome der

Mundhöhle.-Ergebnisse und Konsequenzen 320

Sonderbände

328

Onkologie-Mitteilungen

331

Jahres-Inhalt 1983

340

Autorenverzeichnis 1983 